\title{
Body-centric modelling, identification, and acceleration tracking control of a quadrotor UAV
}

Article

Accepted Version

Alkowatly, M. T., Becerra, V. M. and Holderbaum, W. (2015) Body-centric modelling, identification, and acceleration tracking control of a quadrotor UAV. International Journal of Modelling, Identification and Control, 24 (1). pp. 29-41. ISSN 1746-6172 doi: https://doi.org/10.1504/IJMIC.2015.071697 Available at https://centaur.reading.ac.uk/39735/

It is advisable to refer to the publisher's version if you intend to cite from the work. See Guidance on citing.

Published version at: http://www.inderscience.com/jhome.php?jcode=ijmic

To link to this article DOI: http://dx.doi.org/10.1504/IJMIC.2015.071697

Publisher: Inderscience Publishers

All outputs in CentAUR are protected by Intellectual Property Rights law, including copyright law. Copyright and IPR is retained by the creators or other copyright holders. Terms and conditions for use of this material are defined in the End User Agreement.

www.reading.ac.uk/centaur 
Central Archive at the University of Reading

Reading's research outputs online 


\title{
Body-centric Modelling, Identification, and Acceleration Tracking Control of a Quadrotor UAV
}

\section{Mohamad T. Alkowatly}

School of Systems Engineering,

University of Reading,

Reading, RG6 6AX, UK

E-mail: m.t.alkowatly@pgr.reading.ac.uk

\section{Victor M. Becerra}

School of Systems Engineering,

University of Reading,

Reading, RG6 6AX, UK

E-mail: v.m.becerra@reading.ac.uk

\section{William Holderbaum}

School of Systems Engineering,

University of Reading,

Reading, RG6 6AX, UK

E-mail: w.holderbaum@reading.ac.uk

\begin{abstract}
This paper presents the mathematical development of a body-centric nonlinear dynamic model of a quadrotor UAV that is suitable for the development of biologically inspired navigation strategies. Analytical approximations are used to find an initial guess of the parameters of the nonlinear model, then parameter estimation methods are used to refine the model parameters using the data obtained from onboard sensors during flight. Due to the unstable nature of the quadrotor model, the identification process is performed with the system in closed-loop control of attitude angles. The obtained model parameters are validated using real unseen experimental data. Based on the identified model, a Linear-Quadratic (LQ) optimal tracker is designed to stabilize the quadrotor and facilitate its translational control by tracking body accelerations. The LQ tracker is tested on an experimental quadrotor UAV and the obtained results are a further means to validate the quality of the estimated model. The unique formulation of the control problem in the body frame makes the controller better suited for bio-inspired navigation and guidance strategies than conventional attitude or position based control systems that can be found in the existing literature.
\end{abstract}

Keywords: Quadrotor; Modelling; System Identification; Linear Quadratic Tracking Control.

Reference to this paper should be made as follows:Alkowatly, M.T., Becerra, V.M., and Holderbaum, W. (2014) 'Body-centric Modelling, Identification, and Acceleration Tracking Control of a Quadrotor UAV', International Journal of Modelling, Identification and Control, Vol. x, No. x, pp.xxx-xxx.

Biographical notes: Mohamad T.Alkowatly is a current PhD student in Cybernetics researching visual bio-inspired aerial guidance and navigation strategies. He has an MSc in Aeronautics Engineering (2009, UK) and a BSc in Informatics Engineering (2006, Syria).

Victor M. Becerra is a Professor of Automatic Control. His research interests include computational optimal control, nonlinear control, system identification, robotics, and artificial intelligence.

William Holderbaum is a Professor in Mathematical Engineering. His research interests include boolean input systems, geometric control, rehabilitation engineering, and smart grid systems. 


\section{Introduction}

Quadrotor Unmanned Aerial Vehicles (UAV) have gained considerable interest as a research platform $[1,2,3,4]$. This is because, besides being affordable, they are relatively lightweight and quite manoeuvrable which makes them suitable for testing different flight strategies. This manoeuvrability comes mainly from the fact that quadrotors are unstable in open-loop, and this emphasizes the role of the control system for operating them. This in turn stresses the importance of developing faithful models both for simulation and control design purposes.

In order to obtain a particular model for a specific quadrotor, either the quadrotor dynamics have to be approximated or the model parameters, such as the aerodynamic coefficients and moments of inertia, have to be accurately identified. In the first approach, system identification has been used to identify the attitude angular velocity transfer function of a helicopter [5], thus obtaining a linear and decoupled model of the vehicle. In [6], an Autoregressive structure with External input (ARX) model of a helicopter has been identified by employing neural networks. The second approach, on the other hand, allows to obtain the nonlinear equations of motion of the vehicle by finding or refining the model parameters of a specific quadrotor UAV. It is possible to estimate most of these parameters experimentally as discussed by Derafa et al [7], but that requires the use of a purpose made test bench to hold the UAV and allow appropriate motion and measurements. Alternatively Abas et al. [8] used the unscented Kalman filter to estimate the moments of inertia and the rotor inertia parameters of the quadrotor while the aerodynamic parameters were assumed to be experimentally estimated in advance. However, no action has been taken to validate the resulting model parameters other than the convergence of the unscented Kalman filter, which does not imply that acceptable model parameters have been found. Additionally, extending this method to estimate all model parameters might be limited by their identifiability [9]. Finally the authors in [10] used Prediction Error Methods (PEM) to identify a linear discrete state-space model of a quadrotor UAV. Although such model can be useful for the control design process, the linearity of the model will adversely affect simulation fidelity and restrict its validity to small neighbourhood of the linearisation points.

The first take towards identifying the parameters of a nonlinear model of coaxial helicopter has been presented in [11] where some of the model parameters are found experimentally or analytically while the remaining parameters are estimated using the Covariance Matrix Adaptation Evolution Strategy (CMA-ES). Note that the presence of the stabilizer bar as well as the tandem counter rotating propellers in the coaxial helicopter greatly reduces the rotational dynamics instability if not eliminating it all together. No such mechanical stabilization aid is available on the quadrotor, thus the system identification process has to be adjusted to cope with the fast unstable open loop dynamics of the quadrotor.

Many quadrotor control techniques have been presented in the literature. Most techniques implement separate angular and translational control systems based on a separate models for the rotational and translational dynamics. Simple PID based control systems have been presented $[12,13,14,15]$ where the attitude dynamics are simplified to decouple the attitude angles and then separate feedback loops are used to control each angle. Although the method is reported to work well, ensuring controller stability in all flight conditions is challenging, especially given the use of oversimplified decoupled models.

Sliding mode control of a quadrotor UAVs has been implemented and tested by Öner et al. [16] and Bouabdullah et al. [13]. These two works report contrasting results, so while sliding mode control has been found to provide a very good tracking performance by the former, the latter concluded that the switching nature and the chattering associated with sliding mode control is not suitable for quadrotor control. Backstepping techniques have been used for position tracking in [17], and the Linear Quadratic Regulators (LQR) has been applied for attitude control of quadrotors in [18] where it has provided good stability, but has shown to be slow to react possibly due to modelling uncertainties. Another LQR strategy is demonstrated in [16] providing effective position tracking.

Regardless of the design method used for quadrotor control, all of the aforementioned methods either control the body attitude only or control the translational dynamics through kinematic variables obtained in an inertial frame (position and velocities). Estimating such kinematic variables requires special sensors and rather complicated calculations and filtering schemes. On the other hand, biologically observed visual navigation strategies solve navigation problems without relying on variables in an inertial frame [19, 20, 21, 22, 23], and rely on a body-centric frame of reference instead [24]. Hence, it is more convenient, and more coherent, to develop model based controllers in the same frame of reference. Advantages of employing biologically inspired navigation in UAVs are lighter onboard sensor suites and less computational power requirements [25, 26].

It should be mentioned that it is possible to use the aforementioned conventional control system to serve a bio-inspired navigation method. However, it is more convenient, and more coherent, to develop control systems that do not rely on equivalent variables not normally available in animal species. Furthermore, if the control system is used to validate the applicability of a 
bio-inspired navigation method, it is important not to use variables that the navigation strategy claims not to use. As the control system presented in this paper is used to serve the position and velocity free bio-inspired visual navigation method presented in [27], the control system should not use such variables employed conventionally. Thus, body accelerations are more suitable references for the tracking control system. Attitude tracking control has been assumed to serve as acceleration tracking control in [28]. However, in this research, LQ tracking control design method is used to design an explicit body acceleration tracking control.

This paper demonstrates the modelling, identification and control of a quadrotor helicopter in a body-centric reference frame. A unique feature of this controller is that it provides a means to control the quadrotor translation by tracking body frame accelerations, in contrast to tracking a trajectory defined in an inertial frame. The relation between the the tracked acceleration and the body attitude is analysed. To the authors' knowledge, this paper is the first to address the issues associated with numerical estimation, and validation of the parameters of a nonlinear quadrotor model without requiring any special test benches. The paper provides a numerical quantification of the enhancement achieved by using the estimated model parameters compared to the analytically approximated parameters, and it validates the obtained model parameters using unseen measured data. The body acceleration tracking control system, developed in this paper, is used as an additional validation step for the identified model. Finally, the model and control system presented in this paper are used with the bio-inspired navigation strategy demonstrated in [27].

This paper is organized as follows: The modeling of the quadrotor in a body-centric frame is presented in section 2, estimation of model parameters using analytical approximations is presented in section 3, the formulation of the system identification process is presented in section 4, system identification results and model validation are presented in section 5 , the development of a tracking low level controller based on the proposed model is presented in section 6 , and the results of low level control experimental testing are presented and discussed in section 7 .

\section{Body-Centric Quadrotor Model}

The quadrotor model developed here is similar to that developed in [12]. The model formulation is briefly presented here. Two Cartesian coordinate frames are defined for the purpose of quadrotor modelling. The earth surface fixed frame with axes $\mathbf{1}_{x}^{e}, \mathbf{1}_{y}^{e}$ and $\mathbf{1}_{z}^{e}$ aligned with North, East and Down (NED) local directions respectively, which is safely assumed to be an inertial frame for the purposes of the slow and short flight of the quadrotor. The second frame is a body fixed frame with its origin at the body center of mass and axes $\mathbf{1}_{x}, \mathbf{1}_{y}$ and $\mathbf{1}_{z}$ are aligned with forward, right (starboard), and down body orientations. The body and earth coordinate frames, motor numbering, and their positive rotation direction are illustrated in Figure 1. Note that the axes orientation is different from those used by [12].

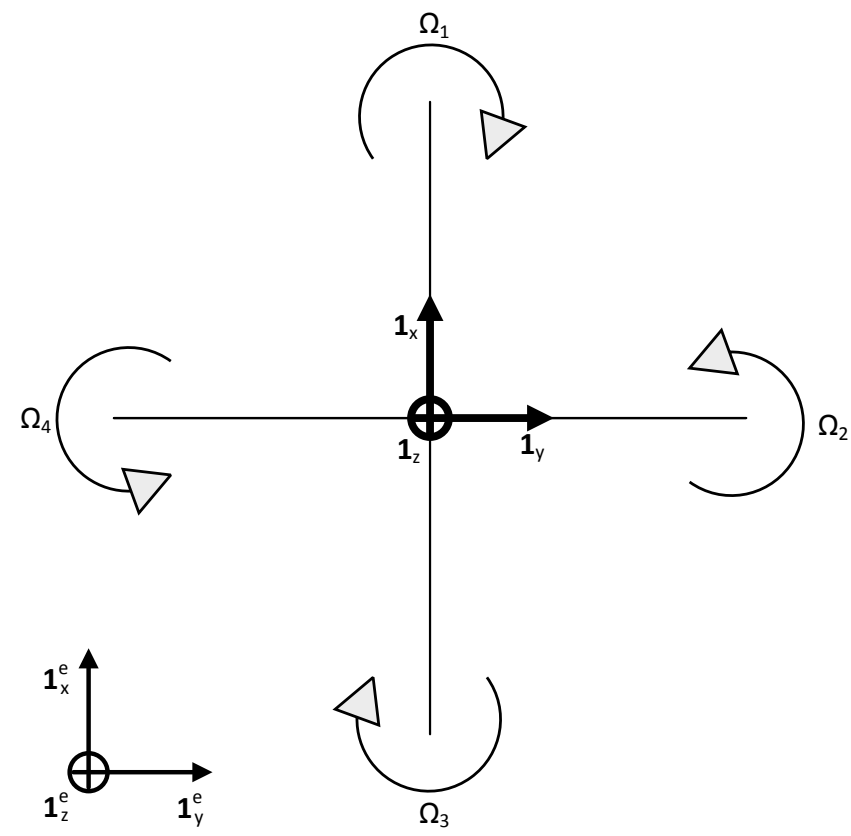

Figure 1 Top view of the quadrotor showing the definition of used coordinates frames, motor numbering and positive motor rotation direction. Axes $\mathbf{1}_{z}^{e}$ and $\mathbf{1}_{z}$ are pointing into the page

\subsection{Attitude and Rotation Representation}

To obtain body attitude starting from the earth frame, one performs three right handed consecutive rotations (yaw, pitch, roll) with angles $(\psi, \theta, \phi)$ about $\mathbf{1}_{z}$, the new $\mathbf{1}_{y}$, and the new $\mathbf{1}_{x}$ axes, respectively. These three rotations define the transformation matrix $R_{b / e}$.

The quadrotor angular velocity in the earth frame $\boldsymbol{\omega}_{b / e}^{e}=[\dot{\phi}, \dot{\theta}, \dot{\psi}]^{T}$ can be found from quadrotor angular velocity in body frame $\boldsymbol{\omega}_{b / e}^{b}=[p, q, r]^{T}$ as follows [29]:

$$
\boldsymbol{\omega}_{b / e}^{e}=\left[\begin{array}{ccc}
1 & \tan (\theta) \sin (\phi) & \tan (\theta) \cos (\phi) \\
0 & \cos (\phi) & -\sin (\phi) \\
0 \sin (\phi) / \cos (\theta) & \cos (\phi) / \cos (\theta)
\end{array}\right] \boldsymbol{\omega}_{b / e}^{b}
$$

\subsection{Quadrotor Body Dynamics}

Using Newton's Euler formalism, the body dynamics are expressed in the body fixed frame as:

$$
\left[\begin{array}{cc}
m \boldsymbol{I}_{3 \times 3} & \mathbf{0}_{3 \times 3} \\
\mathbf{0}_{3 \times 3} & \boldsymbol{I}_{q}
\end{array}\right]\left[\begin{array}{c}
\dot{\boldsymbol{V}}^{b} \\
\dot{\boldsymbol{\omega}}_{b / e}^{b}
\end{array}\right]+\left[\begin{array}{c}
\boldsymbol{\omega}_{b / e}^{b} \times m \boldsymbol{V}^{b} \\
\boldsymbol{\omega}_{b / e}^{b} \times \boldsymbol{I}_{q} \boldsymbol{\omega}_{b / e}^{b}
\end{array}\right]=\left[\begin{array}{c}
\mathbf{F}^{b} \\
\boldsymbol{\tau}^{b}
\end{array}\right]
$$


where $\boldsymbol{I}_{q}$ is the moments of inertia tensor of the quadrotor $\left(\mathrm{kg} \mathrm{m} \mathrm{m}^{2}\right), \boldsymbol{V}^{b}=[u, v, w]^{T}$ is the body frame translational velocity vector $(\mathrm{m} / \mathrm{s}), \boldsymbol{F}^{b}$ is the force vector acting on the quadrotor in body frame $(\mathrm{N})$, and $\boldsymbol{\tau}^{b}$ is the torque vector acting on the quadrotor in body frame $(\mathrm{N}$ $\mathrm{m})$. The quadrotor is assumed to be symmetric about its body principal axes which are assumed to coincide with the body frame. This assumption cancels all products of inertia and the inertia matrix becomes the diagonal $\operatorname{matrix} \boldsymbol{I}_{q}=\operatorname{diag}\left(I_{x x}, I_{y y}, I_{z z}\right)$.

The external forces acting on the quadrotor body are the weight force $(m g)$ and the thrust forces generated by the four propellers $T_{i}$. Each thrust forces is modelled as:

$$
T_{i}=b \Omega_{i}^{2}, i=1,2,3,4
$$

and the total thrust force $T_{a}=T_{1}+T_{2}+T_{3}+T_{4}$ is always aligned with the body $\mathbf{1}_{z}$ axis in the negative direction.

The total torque acting on the quadrotor is composed of the control torques and gyroscopic effect torque. Control torques $\tau_{x}, \tau_{y}$ that generate a positive rolling and pitching moment respectively can be expressed as:

$$
\begin{aligned}
& \boldsymbol{\tau}_{x}=\ell\left(T_{4}-T_{2}\right) \mathbf{1}_{x} \\
& \boldsymbol{\tau}_{y}=\ell\left(T_{1}-T_{3}\right) \mathbf{1}_{y}
\end{aligned}
$$

The aerodynamic drag torque $Q_{i}$ acting on propeller $i$ is modeled as:

$$
Q_{i}=d \Omega_{i}^{2}, i=1,2,3,4
$$

The total drag torque that generates a positive yawing moment is expressed as:

$$
\boldsymbol{\tau}_{z}=d\left(\Omega_{2}^{2}+\Omega_{4}^{2}-\Omega_{1}^{2}-\Omega_{3}^{2}\right) \mathbf{1}_{z}
$$

Body angular rates induce a gyroscopic effect torque $\boldsymbol{\tau}_{r}$ on each of the rotating propellers due to rotor inertia $J_{r}(\mathrm{rad} / \mathrm{s})$, and the total imbalance $\Omega_{r}$ in the propeller angular velocities. $\boldsymbol{\tau}_{r}$ can be expressed as:

$$
\boldsymbol{\tau}_{r}=J_{r}\left(\boldsymbol{\omega}_{b / e}^{b} \times \mathbf{1}_{z}\right) \Omega_{r}=\left[\begin{array}{c}
J_{r} q \Omega_{r} \\
-J_{r} p \Omega_{r} \\
0
\end{array}\right]
$$

where

$$
\Omega_{r}=\Omega_{2}+\Omega_{4}-\Omega_{1}-\Omega_{3}
$$

By defining the following variables:

$$
\begin{aligned}
& U_{1}=\left(\Omega_{1}^{2}+\Omega_{2}^{2}+\Omega_{3}^{2}+\Omega_{4}^{2}\right) \\
& U_{2}=\left(\Omega_{4}^{2}-\Omega_{2}^{2}\right) \\
& U_{3}=\left(\Omega_{1}^{2}-\Omega_{3}^{2}\right) \\
& U_{4}=\left(\Omega_{2}^{2}+\Omega_{4}^{2}-\Omega_{1}^{2}-\Omega_{3}^{2}\right)
\end{aligned}
$$

the quadrotor equations of motion expressed in the body fixed coordinates frame in addition to the local earth attitude kinematics $(\dot{\phi}, \dot{\theta}, \dot{\psi})$ can be written as follows:

$$
\begin{aligned}
& \dot{p}=\left[q r\left(I_{y y}-I_{z z}\right)+J_{r} q \Omega_{r}+\ell b U_{2}\right] / I_{x x} \\
& \dot{q}=\left[p r\left(I_{z z}-I_{x x}\right)-J_{r} p \Omega_{r}+\ell b U_{3}\right] / I_{y y} \\
& \dot{r}=\left[p q\left(I_{x x}-I_{y y}\right)+d U_{4}\right] / I_{z z} \\
& \dot{u}=r v-q w-g \sin (\theta) \\
& \dot{v}=p w-r u+g \cos (\theta) \sin (\phi) \\
& \dot{w}=q u-p v+g \cos (\theta) \cos (\phi)-b U_{1} / m \\
& \dot{\phi}=p+q \tan (\theta) \sin (\phi)+r \tan (\theta) \cos (\phi) \\
& \dot{\theta}=q \cos (\phi)-r \sin (\phi) \\
& \dot{\psi}=q \sin (\phi) / \cos (\theta)+r \cos (\phi) / \cos (\theta)
\end{aligned}
$$

\section{Initial Estimation of Quadrotor Model Parameters}

\subsection{Initial Estimation of Aerodynamic Model Parameters}

Initial Estimation of Lift Factor. The aerodynamic forces and moments generated due to propeller rotation are derived in [30] using the blade element theory. No assumption is made in [30] on the size of the propeller, hence the derivations presented in [30] are valid for larger propellers like the one used in this work. If the ratio of the lateral quadrotor velocities to the propeller's angular velocity is neglected, the lift force generated by the propeller is approximated by:

$$
T=2 \pi \rho N_{b} \bar{c} \Omega^{2} R_{p}^{3}\left[\frac{\theta_{t w_{0}}}{6}-\frac{\theta_{t w_{f}}}{8}-\frac{\lambda}{4}\right]
$$

where $\theta_{t w_{0}}$ and $\theta_{t w_{f}}$ are used to model the propeller variable twist angle at a point $r$ along its radius, which is given by:

$$
\theta_{t w}(r)=\theta_{t w_{0}}-\theta_{t w_{f}}\left(\frac{r}{R_{p}}\right)
$$

Values of $\theta_{t w_{0}}$ and $\theta_{t w_{f}}$ can be found from the propeller geometry using least squares fitting.

From (3) and (11) the lift factor $b$ is expressed as:

$$
b=2 \pi \rho N_{b} \bar{c} R_{p}^{3}\left[\frac{\theta_{t w_{0}}}{6}-\frac{\theta_{t w_{f}}}{8}-\frac{\lambda}{4}\right]
$$

Additionally, if a closed-loop stabilizing controller is available (which is the case in this work), the lift factor $b$ can be estimated experimentally when the quadrotor is hovering, as the total thrust force should be equal to the quadrotor weight. Thus $b$ can also be written as:

$$
b=\frac{m g}{4 \Omega_{h}^{2}}
$$

It is important however to perform this experimental measurement well above the ground to eliminate the contribution of the ground effect. 
Initial estimation of drag factor. The drag torque $Q$ is derived in [30] under the same assumption used for lift force, and can be approximated by:

$$
Q=2 \pi \rho N_{b} \bar{c} \Omega^{2} R_{p}^{4}\left[\frac{C_{d}}{16 \pi}+\lambda\left(\frac{\theta_{t w_{0}}}{6}-\frac{\theta_{t w_{f}}}{8}-\frac{\lambda}{4}\right)\right]
$$

Substituting (15) in (5) and solving for $d$ results in:

$$
d=2 \pi \rho N_{b} \bar{c} R_{p}^{4}\left[\frac{C_{d}}{16 \pi}+\lambda\left(\frac{\theta_{t w_{0}}}{6}-\frac{\theta_{t w_{f}}}{8}-\frac{\lambda}{4}\right)\right]
$$

\subsection{Initial Estimation of the Moments of Inertia}

The estimation of the quadrotor moments of inertia $I_{x x}, I_{y y}, I_{z z}$ about the body axes, and the rotor inertia $J_{r}$ about its rotational axis is simplified by segmenting the quadrotor into simple shapes, working out the individual moments of inertia, and then adding them together [12]. The quadrotor frame is approximated by two rods, the electronics are approximated by a box, the motors are approximated by a cylinder, and the propellers are approximated by a variable density flat cylinder.

\section{System Identification for Refining Model Parameters}

The Prediction Error Method (PEM) has been chosen to estimate the parameters of the nonlinear model (10) due to its ability to provide good estimates even if the data are collected from a closed-loop system [9]. The PEM finds the parameter vector $\Theta$ by using an optimization algorithm to minimize the following error function:

$$
E\left(\boldsymbol{\Theta}, Z_{T}\right)=\frac{1}{N} \sum_{t=1}^{N} \epsilon(\boldsymbol{\Theta}, t)^{T} \boldsymbol{\Lambda} \epsilon(\boldsymbol{\Theta}, t)
$$

where $\boldsymbol{Z}_{T}=\{(u(t), y(t)), t=1, \ldots, N\}$ is the training data set, $\epsilon(\boldsymbol{\Theta}, t)=y(t)-\hat{y}(\boldsymbol{\Theta}, t)$ is the difference between measured and predicted system outputs, $N$ is the total number of time steps in the data, and $\boldsymbol{\Lambda}$ is a diagonal weighting matrix used to reflect the relative importance of the outputs in the minimization process. To prevent the optimization algorithm from being biased in favour variables with large mean values, matrix $\boldsymbol{\Lambda}$ is calculated as shown in (18):

$$
\Lambda=\operatorname{diag}\left(\frac{1}{\bar{y}_{i}^{2}}\right)
$$

where $\bar{y}_{i}=\frac{1}{N} \sum_{i=1}^{N}\left|y_{i}\right|$ is the mean of the absolute value of the output variable $y_{i}$. This will normalize the different variables values for the optimization and will make the error function $E$ (17) unitless, which is useful for comparing results of different data sets as well.
The parameter vector $\Theta$ to be identified is defined as:

$$
\boldsymbol{\Theta}=\left[I_{x x}, I_{y y}, I_{z z}, J_{r}, b, d\right]^{T}
$$

where the initial guess of $\Theta$ is obtained from the analytically approximate values as described in section 3. The search space is constrained within an order of magnitude up and down the initial guess, while three other linear inequality constraints are added to reflect the perpendicular axis theorem constraint on the moment of inertia so that the solution must satisfy:

$$
\begin{aligned}
& I_{x x} \leq I_{y y}+I_{z z} \\
& I_{y y} \leq I_{x x}+I_{z z} \\
& I_{z z} \leq I_{y y}+I_{x x}
\end{aligned}
$$

In principle, the system identification can be performed both in open loop and closed loop configurations. In an open loop configuration, the model inputs are the motor angular velocities $\left(\Omega_{1}\right.$ to $\left.\Omega_{4}\right)$ and the model output is the vehicle measured state $y$ as shown in Figure 2. In the closed loop configuration, a feedback stabilising control system is employed so that the inputs of system model used for identification are the reference commands of the stabilising control $(u)$ and the vehicle measured state $y$ as an output. Although the data is collected from a closed loop system in both cases, the two configuration are considerably different when using the prediction error method to estimate the model parameters. The instability of the open loop model will cause high sensitivity of the error function making the identification process very challenging. This is because the input data, although collected from the closed loop system, will fail to stabilise the unstable quadrotor system in open loop due to the presence of model uncertainties. Therefore the parameter estimation is performed using closed-loop data as this addresses both issues by stabilizing the predictor and reducing the sensitivity of the error function [9]. This is possible since in this work a prior stabilizing controller was available.

The identification experiment is performed in closedloop (Figure 2) by choosing the input vector $\boldsymbol{u}$ to be the remote pilot commands comprising the attitude and throttle reference:

$$
\boldsymbol{u}=\left[\phi_{r}, \theta_{r}, \psi_{r}, \Upsilon_{r}\right]^{T}
$$

while the output vector $\boldsymbol{y}$ is chosen to be:

$$
\boldsymbol{y}=\left[\phi, \theta, \psi, p, q, r, a_{x}, a_{y}, a_{z}\right]^{T}
$$

where body measured accelerations $a_{x}, a_{y}, a_{z}$ are included in the outputs to enhance the observability of the lift factor $b$. These accelerations are given by:

$$
\begin{aligned}
& a_{x}=-g \sin (\theta) \\
& a_{y}=g \cos (\theta) \sin (\phi) \\
& a_{z}=g \cos (\theta) \cos (\phi)-b U_{1} / m
\end{aligned}
$$


The pilot commands references are processed by the on-board closed-loop feedback controller $C_{f b}$ to produce the four motor angular velocities commands $\left[\Omega_{r 1}, \Omega_{r 2}, \Omega_{r 3}, \Omega_{r 4}\right]$ based on the measured quadrotor attitude and attitude rates. Motor commands are then subjected to the actuator dynamics which result in the actual motor angular velocities that drive the model presented in (10). This scheme is illustrated in Figure 2

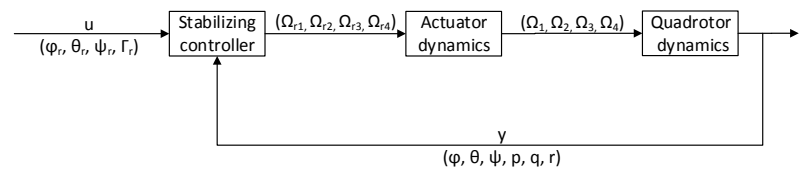

Figure 2 Control scheme used for closed-loop identification of quadrotor system

\section{System Identification Results}

\subsection{Experimental Platform}

Quadrotors are unstable systems that cannot be flown under open loop control. In this work a UAV platform for research by Ascending Technologies GmbH known as the Pelican has been used. The platform comes with an onboard stabilizing controller which is implemented on the on-board ARM7 embedded microcontroller and runs at $1 \mathrm{KHz}$. This controller allows the user to fly the quadrotor by providing attitude reference commands (roll, pitch, and yaw angles) and a throttle value by means of a remote controller. This allows the data acquisition to be performed while flying a closed-loop stable system. Figure 3 shows a side view of the Pelican quadrotor helicopter employed in this work.

Brushless DC motors, like those used by the Pelican, are known to have a small time constant $(10 \mathrm{~ms}$ as reported in [31]). Thus, actuator dynamics are neglected here for simplicity, implying $\left[\Omega_{r 1}, \Omega_{r 2}, \Omega_{r 3}, \Omega_{r 4}\right]=$ $\left[\Omega_{1}, \Omega_{2}, \Omega_{3}, \Omega_{4}\right]$. The supplied stabilizing controller has known structure and parameters, leaving the model parameter vector $\boldsymbol{\Theta}$ in (19) to be estimated by an optimization algorithm.

Output body rates $(p, q, r)$ are provided by onboard Micro Electro Mechanical system (MEMS) gyroscopes while the attitude angles $(\phi, \theta, \psi)$ are estimated by the onboard Attitude and Heading Reference System (AHRS). The body accelerations $\left(a_{x}, a_{y}, a_{z}\right)$ are measured by the onboard MEMS accelerometers, and corrected for gravity using the AHRS attitude estimate. Input and output data are sampled at a rate of $50 \mathrm{~Hz}$ while the quadrotor attitude and throttle is manually

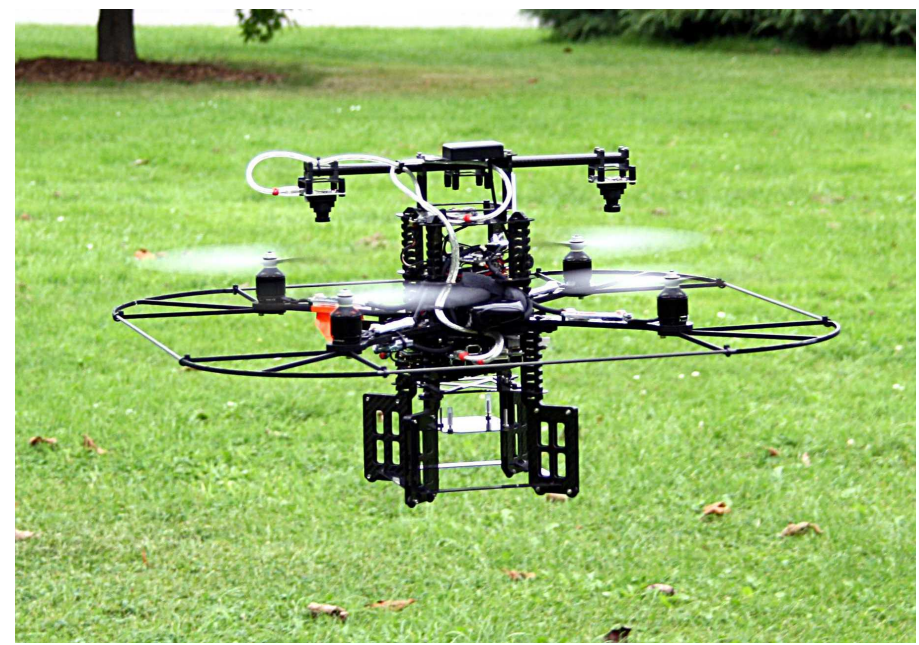

Figure 3 Side view of the Pelican quadrotor helicopter

varied during the experiments. Three experimental data sets, with a duration of 500 seconds each, are collected for the purposes of system identification and model validation.

\subsection{Parameter Estimation}

The initial model parameters values are obtained using the analytical approximations described in section 3, and shown in Table 1 . These values are used as an initial guess for the system identification process, and subsequently refined to achieve more accurate values.

A Quasi-Newton (QN) constrained optimization method from MATLAB's optimization toolbox is used to minimize (17) where the gradients are evaluated numerically and the Hessian is approximated using the BFGS method. A refined estimate of $\Theta$ is found using 500 seconds of real experimental data. Smaller time segments of the experimentally measured against model predicted outputs are shown in Figures 4 and 5 when using the initial guess and the optimized model parameters, respectively. These time segments of the fitted variables have been chosen to show the fitted variables under excitation. A considerable prediction enhancement of $47.61 \%$ is achieved by the optimization process where the error function value after the optimization is $(E=7.67)$ compared to $(E=14.64)$ for the initial guess. The parameters values obtained are listed in Table 1.

\subsection{Validation of The Identified Model}

In order to ensure that the identified model parameters represent well the system dynamics, the model is validated against two data sets of unseen experimental measurements collected from the quadrotor when it was flown manually and all its degrees of freedom were varied. Figure 6 shows different time segments of measured outputs of the validation data set against 


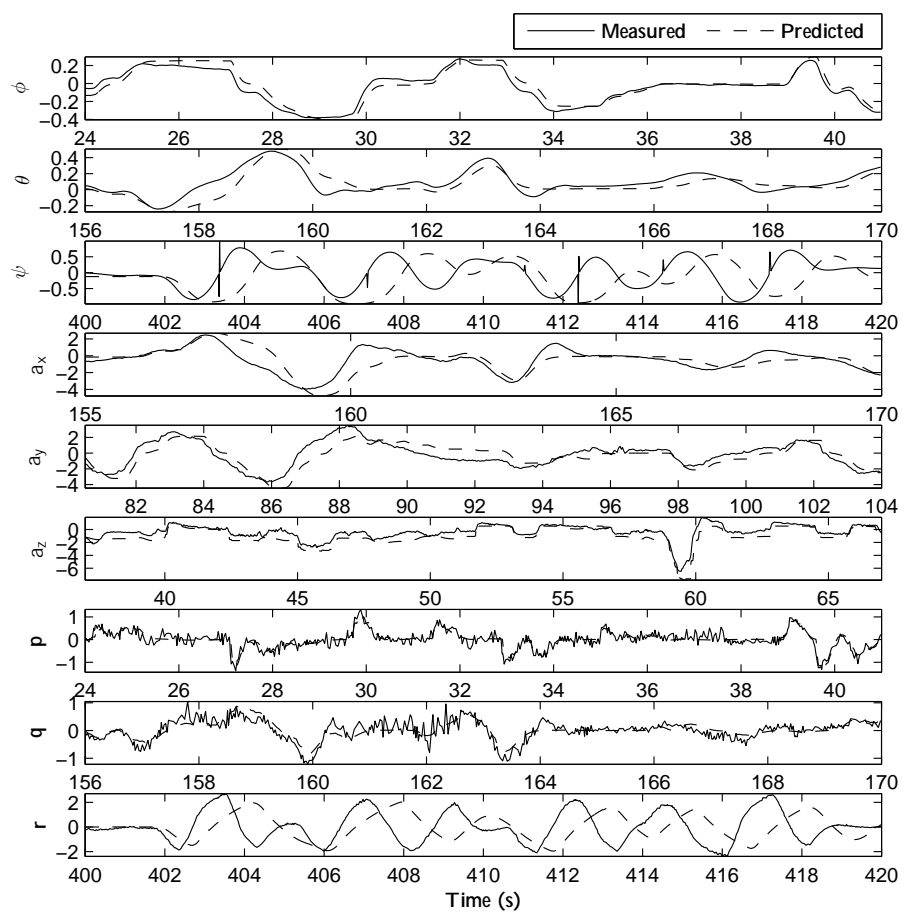

Figure 4 Comparison of model outputs using the initial parameter guesses and the training data set (segments). Angles $(\phi, \theta, \psi)$ are given in radians, accelerations $\left(a_{x}, a_{y}, a_{z}\right)$ are given in $\mathrm{m} / \mathrm{s}^{2}$, and body rates are in $\mathrm{rad} / \mathrm{s}$

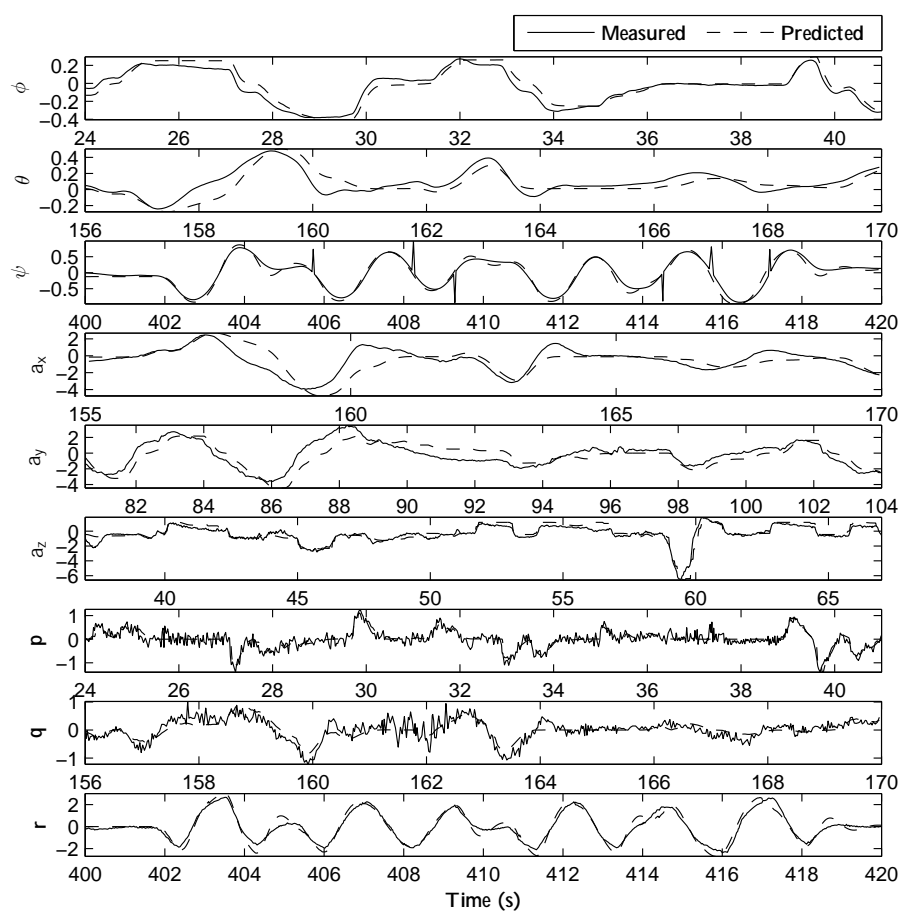

Figure 5 Quasi-Newton Optimization for model output fitting in closed-loop using the training data set (segments). Angles $(\phi, \theta, \psi)$ are given in radians, accelerations $\left(a_{x}, a_{y}, a_{z}\right)$ are given in $\mathrm{m} / \mathrm{s}^{2}$, and body rates are in $\mathrm{rad} / \mathrm{s}$
Table 1 Initial approximations along the system identification refined model parameters

\begin{tabular}{|c|c|c|}
\hline Parameter & Initial value & Refined value \\
\hline$I_{x x}$ & $1.57 \times 10^{-2}$ & $1.21 \times 10^{-2}$ \\
\hline$I_{y y}$ & $1.57 \times 10^{-2}$ & $1.355 \times 10^{-2}$ \\
\hline$I_{z z}$ & $2.52 \times 10^{-2}$ & $2.179 \times 10^{-2}$ \\
\hline$J_{r}$ & $8.031 \times 10^{-5}$ & $1.143 \times 10^{-4}$ \\
\hline$b$ (Analytical) & $2.599 \times 10^{-6}$ & - \\
\hline$b$ (Experimental) & $2.428 \times 10^{-5}$ & $2.256 \times 10^{-5}$ \\
\hline$d$ & $1.247 \times 10^{-7}$ & $3.679 \times 10^{-7}$ \\
\hline \multicolumn{3}{|l}{} \\
\hline
\end{tabular}

the predicted model outputs using the optimized parameters. Additionally, the value of the error function $E$ evaluated when using the initial and the refined model parameters for the training, as well as two unseen data sets are shown in Table 2. There is consistency in the improvement of fitting quality from the initial guess across the training and validation data sets. The model with the optimized parameters is shown to provide about $48 \%$ better fit than the model with the initial parameters values for all the data sets available. This indicates that the model with the identified parameters captures the quadrotor dynamics better than the model with analytically approximated parameters with no signs of over fitting.

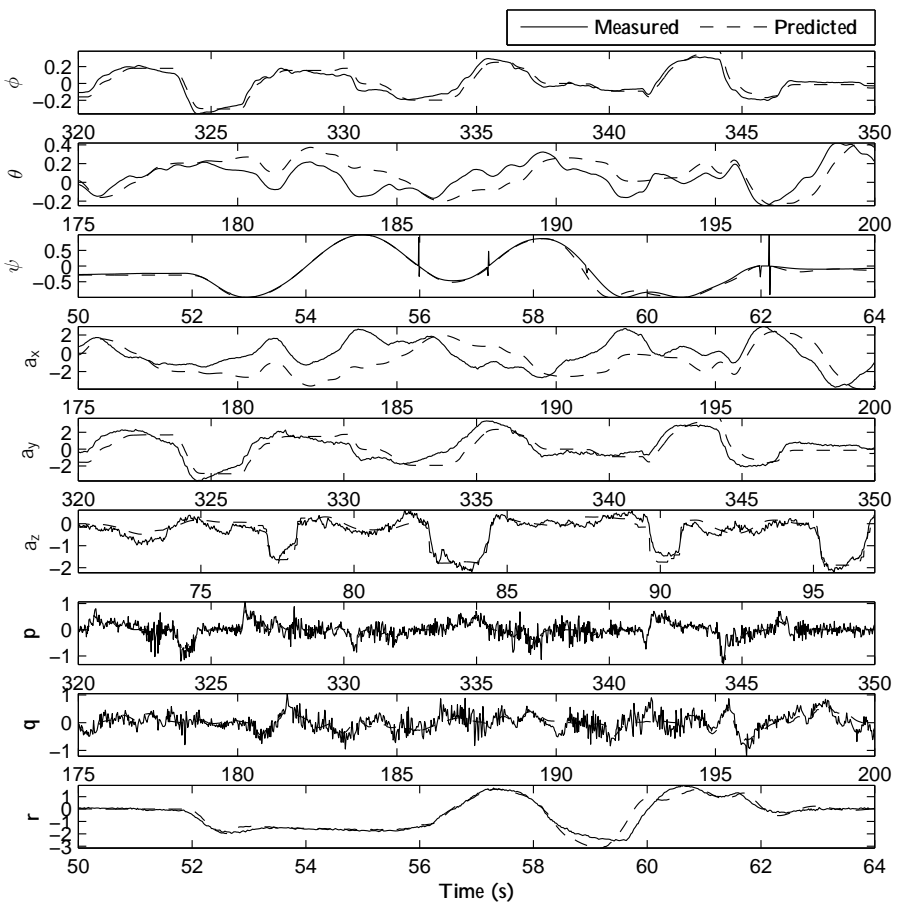

Figure 6 Model validation using one of the unseen data sets (segments). Angles $(\phi, \theta, \psi)$ are given in radians, accelerations $\left(a_{x}, a_{y}, a_{z}\right)$ are given in $\mathrm{m} / \mathrm{s}^{2}$, and body rates are in $\mathrm{rad} / \mathrm{s}$

It is noted that the variable $\theta$ has lower fitting quality compared to other variables both using the training and the validation data sets shown in Figures 
Table 2 Total model fitting error $E$ using initial guess and refined model parameters showing fitting enhancement percentage for each data set

\begin{tabular}{|l|l|l|l|}
\hline Data set & $\begin{array}{l}\text { Using } \\
\text { initial } \\
\text { guess }\end{array}$ & $\begin{array}{l}\text { Using } \\
\text { refined } \\
\text { parameters }\end{array}$ & $\begin{array}{l}\text { Model } \\
\text { fitting } \\
\text { enhancement }\end{array}$ \\
\hline $\begin{array}{l}\text { Training } \\
\text { data set }\end{array}$ & 14.64 & 7.67 & $47.61 \%$ \\
\hline $\begin{array}{l}\text { Unseen } \\
\text { data set 1 }\end{array}$ & 18.93 & 8.71 & $48.59 \%$ \\
\hline $\begin{array}{l}\text { Unseen } \\
\text { data set 2 }\end{array}$ & 16.81 & 8.79 & $47.72 \%$ \\
\hline
\end{tabular}

5 and 6 , respectively. Consequently, the variable $a_{x}$ shows similar quality as it is directly related to $\theta$. The same results have been found even when increasing the weight corresponding to those variables in the weighting matrix $\boldsymbol{\Lambda}$. This indicates that there is a trade off between fitting $\theta$ and $a_{x}$, and fitting all other variables in order to minimize the total error function $E$ (17). The relative inaccuracy in fitting $\theta$ may be explained by minor violations of modelling assumptions, including for instance the presence of an small imbalance in the center of mass on the $\mathbf{1}_{y}$ quadrotor axis.

Nevertheless, the fitting of the corresponding body rate $q$ is acceptable, although still more sluggish than other rates, which explains the observed lag between the predicted and measured values of $\theta$. Note that the prediction error of $\theta$ is bounded for all the data sets available $\left(L_{\infty}(\theta-\hat{\theta})<0.58\right)$.

As a final remark, despite the good prediction accuracy achieved by performing the identification in closed-loop, it has been found that the best model parameters achieved fail to predict the quadrotor dynamics for more than 2 seconds if prediction is performed in open loop. This is due to the unstable nature of the open loop system.

\section{Quadrotor Low Level Control}

In this section the design of a control system is presented based on the body-centric model of the quadrotor dynamics (10). To fit within a biologically inspired navigation scheme, it is desired to develop a biologically plausible low-level control. The definition of a biologically plausible control system has different interpretations from different points of view (neurological or ecological). Moreover, the factors that make a control system biologically plausible are different depending on the animal species used as reference. In this work, the guidelines for biological plausibility suggested in [24] are followed. Hence, a biologically plausible control should only use the information equivalent to that provided by sensory organs such as the vestibular system. Additionally, the system should use this information in the body-centric fame of reference which is the motive to develop the model (10) in the body frame.

Functionally, the desired control system must be able to stabilize the fast quadrotor rotational dynamics in addition to facilitate the control of the quadrotor translational dynamics by a higher level controller without relying on kinematic variables that are defined in an external reference, such as position or velocity. The attitude reference, particularly the roll and pitch angles, are an exception because they are defined by the gravity vector and body shape, both of which are perceived by many animal species.

Based on the previous guidelines, and noting the system dynamic equations (10), the body velocities $u, v, w$ do not affect the rest of the states and can be dropped from the state vector for the purposes of control synthesis. The state vector $\boldsymbol{x}$ then becomes:

$$
\boldsymbol{x}=[\phi, \theta, \psi, p, q, r]^{T}
$$

the control vector $\boldsymbol{u}$ is

$$
\boldsymbol{u}=\left[\Omega_{1}, \Omega_{2}, \Omega_{3}, \Omega_{4}\right]^{T}
$$

and the output vector $\boldsymbol{y}$ is

$$
\boldsymbol{y}=\left[\psi, a_{x}, a_{y}, a_{z}\right]^{T}
$$

where the body accelerations $a_{x}, a_{y}, a_{z}$, which depend on the states and the input as shown in (24), are assumed to be available through the on-board accelerometers. On the other hand, the heading angle $\psi$ is only available through an external reference (like the earth magnetic field or an arbitrarily chosen heading reference) and it is included in the state and output vectors only to aid testing and flying the quadrotor by a human operator. It is clear from the model equations (10) that dropping the heading angle dynamics $\psi$ has no effect whatsoever on the quadrotor dynamics in the body frame, in contrast with the case when the model is derived in an inertial frame [13].

The dynamic equations (now a subset of (10)) are linearised about the equilibrium point $\left(\boldsymbol{x}_{e q}, \boldsymbol{u}_{e q}\right)$ given by:

$$
\boldsymbol{x}_{e q}=[0,0,0,0,0,0]^{T} \quad \boldsymbol{u}_{e q}=\left[\Omega_{h}, \Omega_{h}, \Omega_{h}, \Omega_{h}\right]^{T}
$$

where $\Omega_{h}=407 \mathrm{rad} / \mathrm{s}$ for the Pelican. The linearised equations are discretised to obtain the system matrices $\boldsymbol{\Phi}, \boldsymbol{\Gamma}, \boldsymbol{C}, \boldsymbol{D}$ of a linear discrete state space representation of the quadrotor system:

$$
\begin{aligned}
\boldsymbol{x}(n+1) & =\boldsymbol{\Phi} \boldsymbol{x}(n)+\boldsymbol{\Gamma} \boldsymbol{u}(n) \\
\boldsymbol{y}(n) & =\boldsymbol{C} \boldsymbol{x}(n)+\boldsymbol{D} \boldsymbol{u}(n)
\end{aligned}
$$

The values of the system matrices $\boldsymbol{\Phi}, \boldsymbol{\Gamma}, \boldsymbol{C}, \boldsymbol{D}$ are shown in the appendix. 


\subsection{Optimal Tracker Control Design}

To stabilize the rotational dynamics in addition to controlling the translational dynamics of the quadrotor, the following control law is used:

$$
\boldsymbol{u}(n)=-\boldsymbol{K} \boldsymbol{x}(n)+\boldsymbol{F} \boldsymbol{y}_{r}(n+1)
$$

where matrices $\boldsymbol{K}, \boldsymbol{F}$ are the state feedback and reference feed-forward gains respectively. The rotational dynamics are stabilized due to the state feedback term in (30). To allow the tracking of desired translational reference, the output reference $\boldsymbol{y}_{r}$ is defined as :

$$
\boldsymbol{y}_{r}=\left[\psi_{r}, a_{x r}, a_{y r}, a_{z r}\right]^{T}
$$

The references for body accelerations $a_{x r}, a_{y r}, a_{z r}$ enable the translational control of the quadrotor along the three body axes, while the yaw angle reference $\psi_{r}$ allows the operator to control the quadrotor's heading. As this lowlevel control is designed to be used by a high level bioinspired navigation control system, then the low-level controller is chosen to be simple as high performance is not required from it.

Linear Quadratic (LQ) optimal control methods have been used to obtain the values of $\boldsymbol{K}$ and $\boldsymbol{F}$ in (30). Equation (24) shows that the system has direct feedthrough of the control input in the output acceleration equations (resulting in a non-zero $\boldsymbol{D}$ matrix), thus the gain matrices have been found using the formulation presented in [32] which assumes a constant reference signal and involves the following performance index:

$J=\frac{1}{2} \sum_{n=0}^{\infty}\left\{\left(\boldsymbol{y}(n)-\boldsymbol{y}_{r}\right)^{T} \boldsymbol{Q}_{y}\left(\boldsymbol{y}(n)-\boldsymbol{y}_{r}\right)+\boldsymbol{u}(n)^{T} \boldsymbol{R} \boldsymbol{u}(n)\right\}$

The obtained values of $\boldsymbol{K}$ and $\boldsymbol{F}$ are shown in the appendix. Lastly, the overall control scheme is illustrated in Figure 7.

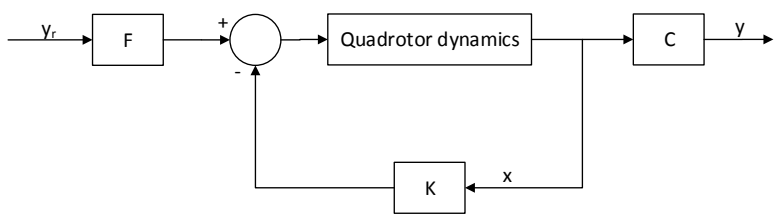

Figure 7 LQ Tracker controller structure for low-level control

\subsection{Tuning and Analysis}

A Simulink model is developed implementing the control structure shown in Figure 7 using the nonlinear model
(10). The references are provided externally by the user via a joystick. Initial tuning of the controller weight matrices $\boldsymbol{Q}_{y}$ and $\boldsymbol{R}$ was performed in simulation and further tuning was performed experimentally. The final chosen values of weight matrices are given in the appendix.

The LQ tracker provides sub-optimal tracking due to the linearisation of the non-linear quadrotor dynamics in addition to unsatisfied assumptions in the optimality problem, such as the constant reference value. Besides achieving sub-optimal tracking, LQ optimal control design methods guarantee nominal stability under mild assumptions[33].

It is useful to study the effect of tracking acceleration commands on the system states. When the controller is presented with a zero reference signal to track, the feedback term in control law (30) will stabilize the quadrotor about its original equilibrium point $\boldsymbol{x}_{e q}=\mathbf{0}$. However, when a non-zero reference signal $\boldsymbol{y}_{r}$ is presented, the feed-forward term will displace the equilibrium point at steady state to $\boldsymbol{x}_{s s}$. This state can be found from the system dynamics equations (29) when the control law (30) is used to produce the control input vector $\boldsymbol{u}$. Substituting (30) in (29) and dropping the time dependency results in:

$$
\boldsymbol{x}_{s s}=\boldsymbol{\Phi} \boldsymbol{x}_{s s}+\boldsymbol{\Gamma}\left(-\boldsymbol{K} \boldsymbol{x}_{s s}+\boldsymbol{F} \boldsymbol{y}_{r}\right)
$$

solving for $\boldsymbol{x}_{s s}$ gives:

$$
\boldsymbol{x}_{s s}=\Pi \boldsymbol{y}_{r}
$$

where $\Pi$ is given by:

$$
\boldsymbol{\Pi}=(\boldsymbol{I}-\boldsymbol{\Phi}+\boldsymbol{\Gamma} \boldsymbol{K})^{-1} \boldsymbol{\Gamma} \boldsymbol{F}
$$

and $\boldsymbol{I}$ is the identity matrix of the same dimensions as $\boldsymbol{\Phi}$. Using the parameter values shown in the appendix, matrix $(\boldsymbol{I}-\boldsymbol{\Phi}+\boldsymbol{\Gamma} \boldsymbol{K})$ is invertible and the numerical value of $\Pi$ is shown in the appendix.

Inspecting the value of $\boldsymbol{\Pi}$ reveals an interesting property. Firstly, positive longitudinal body acceleration $a_{x r}$ is tracked by demanding a negative pitch angle while positive lateral body acceleration $a_{y r}$ is tracked by demanding a positive roll angle. Thus, one can limit the maximum attitude angles (at steady state) by limiting the values of the demanded acceleration reference. Limiting $a_{x r}$ and $a_{y r}$ to values in the range $\left[-\bar{a}_{r}, \bar{a}_{r}\right]$ results in a maximum pitch and roll angles of $0.102 \bar{a}_{r} \mathrm{rad}$. This maximum value is not exact if the reference is not constant and because of nonlinearities in the dynamics. However, $\bar{a}_{r}$ can be set to provide a safe operation of the vehicle and to prevent driving the attitude angles too far from the point the nonlinear model was linearised at. Finally, the motors cannot change their spin direction, and hence cannot generate a negative thrust. Therefore, the maximum feasible downward acceleration cannot exceed the value of free fall gravity acceleration of $9.805 \mathrm{~m} / \mathrm{s}$. 


\section{Low Level Control Experimental Results and Discussion}

The control system presented in the previous section is implemented on the Pelican ARM embedded processor which runs the control loop at a frequency of $1 \mathrm{KHz}$. The state vector $\boldsymbol{x}$ is estimated by the onboard Attitude and Heading Reference System (AHRS) while the output reference vector $\boldsymbol{y}_{r}$ is provided by the remote controller. The quadrotor is flown indoors and both the commanded body accelerations $a_{x r}, a_{y r}, a_{z r}$ as well as the measured body accelerations corrected for gravity $a_{x}, a_{y}, a_{z}$ are logged at a rate of $50 \mathrm{~Hz}$. Figure 8 shows 50 seconds of the experimental data showing the reference signals against measured body accelerations.

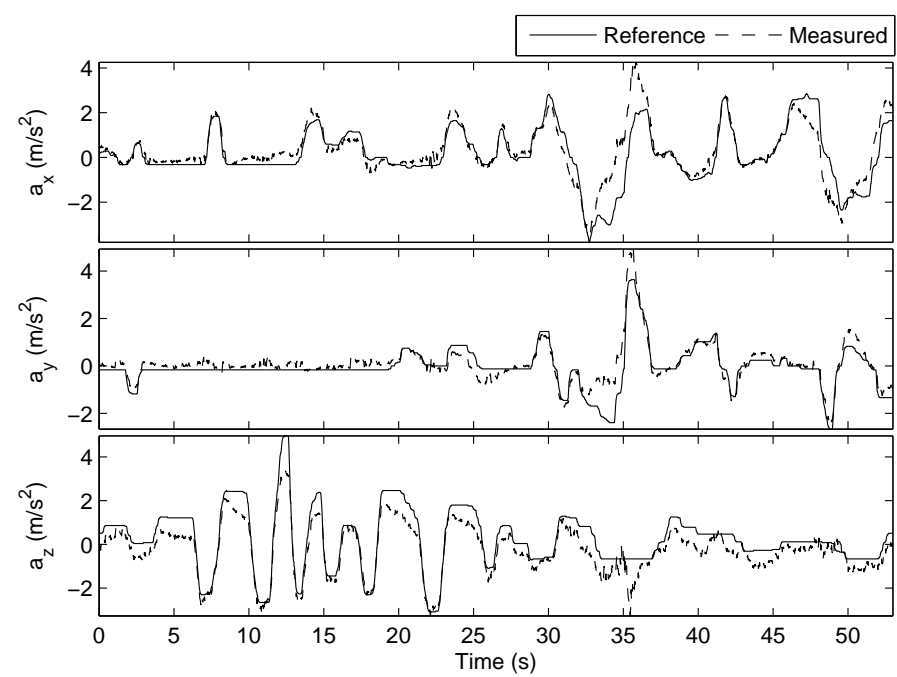

Figure 8 Reference vs. actual body accelerations (segment).

The proposed controller managed to achieve its aim of stabilizing the quadrotor attitude and providing a mean to control the translational dynamics. In addition to the smooth and stable flight achieved experimentally, it can be seen that the tracking performance of body acceleration shown in Figure 8 is very satisfactory. The previous sentence has to be interpreted taking into account the limitations of the linearised control and the feed forward acceleration tracking employed. As the quadrotor dynamics (10) and output acceleration equations (24) are linearised around zero attitude angles, then the attitude contribution to changes in the body accelerations are not fully captured. This becomes evident by comparing the tracking performance of small accelerations in the first 10 seconds of Figure 8 compared to simultaneous, larger, and more sustained accelerations around second 35 in the same figure. Another artefact of linearising the system dynamics can be seen on the vertical body acceleration $a_{z}$ which shows a tendency to track upward (negative) reference accelerations better than downward accelerations as shown in Figure 8. This is mainly due to linearising the nonlinear relation of the thrust force with respect to the motor angular velocity (11).

In addition to the linearisation limitations, it should be noted that the acceleration tracking results shown have been achieved without an acceleration feedback loop correcting for any observed errors. Nevertheless, the tracking performance obtained was satisfactory especially given that the proposed control system will act under the higher-level visual control presented in [27], where the visual variables of interest will be under a feedback control cascaded with the control system presented here. The tracking performance presented in Figure 8 has another use in the context of this paper as the tracking performance is partly a measure of the quality of the linearised model employed, which is derived from the identified nonlinear model.

Another experiment is performed where individual body accelerations have been demanded separately to reduce the attitude coupling effect on the output acceleration, which in not fully captured by the linearised model. Figure 9 shows 3 different time segments of the experiment each showing one of the body accelerations being excited where the reference and measured body accelerations are plotted. Achieving fairly accurate tracking performance as shown in Figure 9 reflects the quality of the model parameters obtained in this paper. This can be considered an additional validation step in addition to performing the unseen data fitting test as well as the experimental control of the quadrotor under a model based control system. Additionally, these results show that despite the relative difference in the fitting quality of variables $\theta$ and $a_{x}$ compared to the other variables has no noticeable effects on the quality of the tracking of longitudinal and lateral accelerations as shown in Figures 8 and 9. Therefore, it is possible to conclude with confidence that the proposed model structure and parameter estimation methods employed resulted in a nonlinear model of the quadrotor that is sufficiently accurate for the purpose of control system design.

\section{Conclusions}

In this paper, a body-centric non-linear model for a quadrotor UAV has been presented, identified, verified and used in a model based body acceleration tracking control system. The proposed approach is an attractive choice for researchers investigating bioinspired navigation strategies for quadrotor UAVs. The parameters of the nonlinear model are initially approximated by analytical methods and then further refined using system identification methods. Due to the unstable nature of the quadrotor model the system identification is performed in closed loop to avoid the issues associated with the integration of unstable 


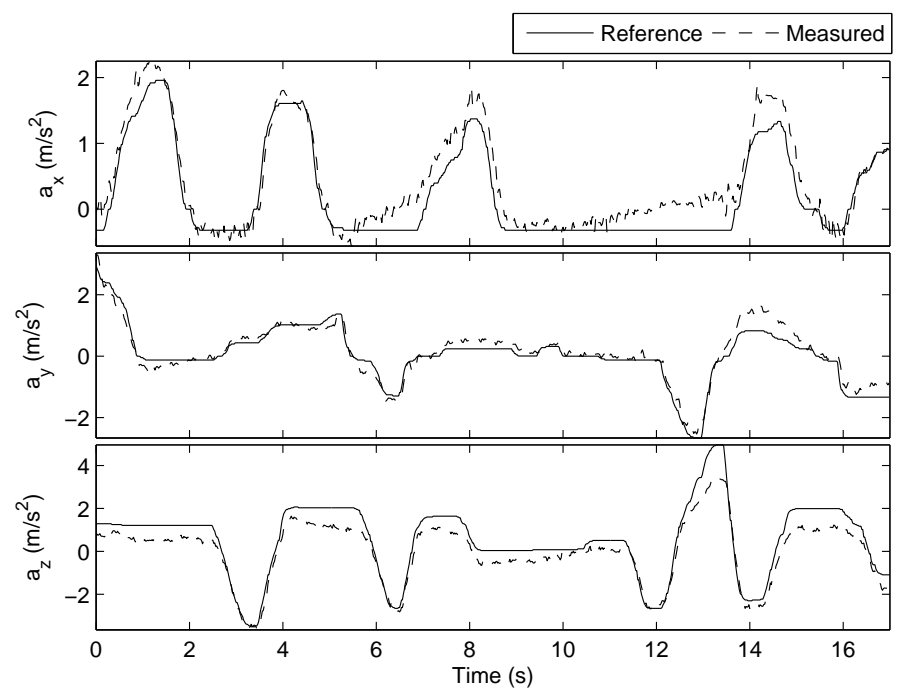

Figure 9 Reference vs. actual body accelerations when no two accelerations are demanded simultaneously

dynamics and the high sensitivity of the error function. Performing the identification in closed loop resulted in satisfactory prediction of the quadrotor dynamics when using sets of unseen experimental data.

The results obtained with the proposed control method demonstrate the possibility of using bodycentric based control to operate the quadrotor, which is more suitable for fulfilling bio-inspired navigation strategies. The proposed control system has been tested experimentally and it performed sufficiently well in stabilizing the quadrotor and controlling its translation. The good tracking performance achieved via the feedforward tracking is another direct indication of the good quality of the model achieved via the system identification process carried out in this paper. The body-centric model and the body acceleration tracking control proposed in this paper are the building blocks of the visual high-level control and state estimation presented in [27].

\section{References}

[1] Vijay Kumar and Nathan Michael. Opportunities and challenges with autonomous micro aerial vehicles. The International Journal of Robotics Research, 31(11):1279-1291, September 2012.

[2] R Ritz, M W Muller, M Hehn, and R D'Andrea. Cooperative quadrocopter ball throwing and catching, 2012.

[3] Daewon Lee, Hyon Lim, H Jin Kim, Youdan Kim, and Kie Jeong Seong. Adaptive Image-Based Visual Servoing for an Underactuated Quadrotor System. Journal of Guidance, Control, and Dynamics, 35(4):1335-1353, July 2012.
[4] Stephan Weiss, Markus W Achtelik, Simon Lynen, Michael C Achtelik, Laurent Kneip, Margarita Chli, and Roland Siegwart. Monocular Vision for Longterm Micro Aerial Vehicle State Estimation: A Compendium. Journal of Field Robotics, 30(5):803831, 2013.

[5] Jin-hua Wang, Zhi-qiang Bai, and Pei-zhi Liu. Modelling and control system design of a smallscale unmanned helicopter. International Journal of Modelling, Identification and Control, 12(1):12-16, 2011.

[6] Syariful S Shamsudin and XiaoQi Chen. Identification of an unmanned helicopter system using optimised neural network structure. International Journal of Modelling, Identification and Control, 17(3):223-241, 2012.

[7] L Derafa, T Madani, and A Benallegue. Dynamic Modelling and Experimental Identification of Four Rotors Helicopter Parameters. In Industrial Technology, 2006. ICIT 2006. IEEE International Conference on, pages 1834-1839, 2006.

[8] N Abas, A Legowo, and R Akmeliawati. Parameter identification of an autonomous quadrotor. In Mechatronics (ICOM), 2011 4th International Conference On, pages 1-8, 2011.

[9] L Ljung. System identification. Wiley Online Library, 1999.

[10] I Stanculeanu and T Borangiu. Quadrotor BlackBox System Identification. World Academy of Science, Engineering and Technology, (0054), 2011.

[11] D Schafroth, C Bermes, S Bouabdallah, and R Siegwart. Modeling, system identification and robust control of a coaxial micro helicopter. Control Engineering Practice, 18(7):700-711, July 2010.

[12] T Bresciani. Modelling, identification and control of a quadrotor helicopter. Master thesis, Lund University, 2008.

[13] Samir Bouabdallah and Roland Siegwart. Design and control of quadrotors with application to autonomous flying. $\mathrm{PhD}$ thesis, 2007.

[14] Huang Haomiao, G M Hoffmann, S L Waslander, and $\mathrm{C}$ J Tomlin. Aerodynamics and control of autonomous quadrotor helicopters in aggressive maneuvering. In Robotics and Automation, 2009. ICRA '09. IEEE International Conference on, pages 3277-3282, 2009.

[15] A L Salih, M Moghavvemi, H A F Mohamed, and K S Gaeid. Modelling and PID controller design for a quadrotor unmanned air vehicle. In Automation Quality and Testing Robotics (AQTR), 2010 IEEE International Conference on, volume 1, pages 1-5, 2010. 
[16] Kaan Taha Öner, Ertuğrul Çetinsoy, Efe Sırımoğlu, Cevdet Hancer, Taylan Ayken, and Mustafa Ünel. LQR and SMC stabilization of a new unmanned aerial vehicle. In International Conference on Intelligent Control, Robotics, and Automation (ICICRA09), Venice, Italy, 2009. World Academy of Science, Engineering and Technology.

[17] T Madani and A Benallegue. Backstepping Control for a Quadrotor Helicopter. In Intelligent Robots and Systems, 2006 IEEE/RSJ International Conference on, pages 3255-3260, 2006.

[18] S Bouabdallah, A Noth, and R Siegwart. PID vs LQ control techniques applied to an indoor micro quadrotor. In Intelligent Robots and Systems, 2004. (IROS 2004). Proceedings. 2004 IEEE/RSJ International Conference on, volume 3, pages 24512456 vol.3, 2004.

[19] M Srinivasan, S Zhang, M Lehrer, and T Collett. Honeybee navigation en route to the goal: visual flight control and odometry. Journal of Experimental Biology, 199(1):237-244, 1996.

[20] D N Lee, M N O Davies, and P R Green. Visual control of velocity of approach by pigeons when landing. Journal of Experimental Biology, 180(1):85-104, 1993.

[21] D Izzo, N Weiss, and T Seidl. Constant-Optic-Flow Lunar Landing: Optimality and Guidance. Journal of Guidance Control and Dynamics, 34(5):1383, 2011.

[22] Dario Izzo and Guido De Croon. Landing with Time-to-Contact and Ventral Optic Flow Estimates. Journal of Guidance, Control, and Dynamics, 35(4):1362-1367, July 2012.

[23] Herisse, X, B., T Hamel, R Mahony, and F X Russotto. Landing a VTOL Unmanned Aerial Vehicle on a Moving Platform Using Optical Flow. IEEE Transactions on Robotics, 28(1):77-89, 2012.

[24] J H Evers. Biological inspiration for agile autonomous air vehicles. Technical report, 2007.

[25] Antoine Beyeler, Jean-Christophe Zufferey, and Dario Floreano. Vision-based control of nearobstacle flight. Autonomous Robots, 27(3):201-219, 2009.

[26] Nicolas Franceschini, Franck Ruffier, Julien Serres, and Stéphane Viollet. Optic flow based visual guidance: from flying insects to miniature aerial vehicles. Aerial vehicles, 35:747, 2009.

[27] Mohamad T Alkowatly, Victor M Becerra, and William Holderbaum. Bio-inspired Autonomous Visual Vertical Control of A Quadrotor UAV. Journal of Guidance, Control, and Dynamics, 2014. Accepted, In press.
[28] B Herisse, F X Russotto, T Hamel, and R Mahony. Hovering flight and vertical landing control of a VTOL Unmanned Aerial Vehicle using optical flow. In Intelligent Robots and Systems, 2008. IROS 2008. IEEE/RSJ International Conference on, pages 801-806, 2008.

[29] Brian L Stevens and Frank L Lewis. Aircraft control and simulation. Wiley New York, 2 edition, 2003.

[30] G Fay. Derivation of the aerodynamic forces for the mesicopter simulation. Technical report, Stanford University, California, USA, 2001.

[31] O Moseler and $\mathrm{R}$ Isermann. Application of model-based fault detection to a brushless DC motor. Industrial Electronics, IEEE Transactions on, 47(5):1015-1020, 2000.

[32] J H Wang. An Active Low-Order Fault-Tolerant State Space Self-Tuner for the Unknown SampleData Linear Regular System with an Input-Output Direct Feedthrough Term. Applied mathematical sciences, 6(97):4813, 2012.

[33] F L Lewis, D Vrabie, and V L Syrmos. Optimal control. Wiley, 2012.

\section{Appendix}

Table A1: Discrete time linearised model and LQ tracker parameters

\begin{tabular}{|c|c|c|c|c|}
\hline$\Phi$ & & {$\left[\begin{array}{lllllc}1 & 0 & 0 & 0.001 & 0 \\
0 & 1 & 0 & 0 & 0.001 \\
0 & 0 & 1 & 0 & 0 \\
0 & 0 & 0 & 1 & & 0 \\
0 & 0 & 0 & 0 & & 1 \\
0 & 0 & 0 & 0 & & 0\end{array}\right.$} & $\left.\begin{array}{c}0 \\
0 \\
0.001 \\
0 \\
0 \\
1\end{array}\right]$ & \\
\hline$\Gamma$ & $10^{-3} \times$ & $\begin{array}{cc}1 & -0.0002 \\
0.0001 & 0 \\
0 & 0 \\
0 & -0.3305 \\
0.295 & 0 \\
-0.0143 & 0.0143\end{array}$ & $\begin{array}{c}0 \\
-0.0001 \\
0 \\
0 \\
-0.295 \\
-0.0143\end{array}$ & $\left.\begin{array}{c}0.0002 \\
1 \\
0 \\
0 \\
0.3305 \\
0 \\
0.0143\end{array}\right]$ \\
\hline$C$ & & $\begin{array}{ccc}0 & 0 & 1 \\
0 & -9.805 \\
9.805 & 0 & 0 \\
0 & 0 & 0\end{array}$ & $\left.\begin{array}{llll}1 & 0 & 0 & 0 \\
0 & 0 & 0 & 0 \\
0 & 0 & 0 & 0 \\
0 & 0 & 0 & 0\end{array}\right]$ & \\
\hline
\end{tabular}


Table A1 (continued)

\begin{tabular}{|c|c|}
\hline$D$ & {$\left[\begin{array}{cccc}0 & 0 & 0 & 0 \\
0 & 0 & 0 & 0 \\
0 & 0 & 0 & 0 \\
-0.0116 & -0.0116 & -0.0116 & -0.0116\end{array}\right]$} \\
\hline$K$ & $\begin{array}{cccccc}0 & 530.32 & -211.61 & 0 & 42.4 & -86.12 \\
-529.93 & 0 & 211.61 & -40.04 & 0 & 86.12 \\
0 & -530.32 & -211.61 & 0 & -42.4 & -86.12 \\
529.93 & 0 & 211.61 & 40.04 & 0 & 86.12\end{array}$ \\
\hline $\boldsymbol{F}$ & {$\left[\begin{array}{cccr}-211.61 & -54.09 & 0 & -21.52 \\
211.61 & 0 & -54.05 & -21.52 \\
-211.61 & 54.09 & 0 & -21.52 \\
211.61 & 0 & 54.05 & -21.52\end{array}\right]$} \\
\hline $\boldsymbol{Q}_{y}$ & $10^{3} \times\left[\begin{array}{cccc}180 & 0 & 0 & 0 \\
0 & 5 & 0 & 0 \\
0 & 0 & 5 & 0 \\
0 & 0 & 0 & 5\end{array}\right]$ \\
\hline $\boldsymbol{R}$ & $\boldsymbol{I}_{4}$ \\
\hline$\Pi$ & {$\left[\begin{array}{cccc}0 & 0 & 0.102 & 0 \\
0 & -0.102 & 0 & 0 \\
1 & 0 & 0 & 0 \\
0 & 0 & 0 & 0 \\
0 & 0 & 0 & 0 \\
0 & 0 & 0 & 0\end{array}\right]$} \\
\hline
\end{tabular}

